\title{
Guanine Nucleotide-Binding Protein G(s) Subunit Alpha Isoforms XLas
}

National Cancer Institute

\section{Source}

National Cancer Institute. Guanine Nucleotide-Binding Protein G(s) Subunit Alpha

Isoforms XLaS. NCI Thesaurus. Code C17983.

Guanine nucleotide-binding protein G(s), subunit alpha isoforms XLas (1037 aa, 111 kDa) is encoded by the human GNAS gene. This protein may play a role in signal transduction by activating adenylate cyclase in response to beta-adrenergic stimuli. 